\section{Aktuelle Fragen zur Rindfleischerzeugung unter besonderer Berücksichtigung der Haltung von Mutterkühen}

\author{
Wilhelm Neumann \\ Geschäftsführer der EZG für Rinder und Schweine Nordost \\ $\mathrm{GmbH}$, ehemals Universität Rostock
}

In der EU ist die Rindfleischerzeugung nur im Zusammenhang mit der Milchrind - u. Fleischrindproduktion zu sehen.

In M/V haben sich die Rinder - u. Kuhbestände seit 1991 so entwickelt, wie in der Tabelle dargestellt (Tabelle 1).

Die Tendenz gilt in etwa für alle 5 neuen Bundesländer. Während die Zahl der Milchkühe auf etwa $75 \%$ gesunken ist, stieg die der Mutterkühe auf $660 \%$ an; ist aber in der absoluten Zahl noch bedeutend geringer.

Gleichzeitig hat die Milchmengenleistung der Kühe durch kontinuierlich Steigerung eine respektable Höhe erreicht (Tabelle 2).

Daraus ist zu entnehmen, dass die kontrollierten Kühe in den fünf neuen Bundesländern beachtlich über dem Gesamtdurchschnitt liegen. Besser sind nur Niedersachen mit $7.963 \mathrm{~kg}$, Weser - Ems mit 7.812 kg und Westfalen mit 7.742 kg je Kuh.

Die quotierte Milch wird demzufolge mit immer weniger Kühen produziert, die Zahl der Milchkühe geht zurück und Grünlandflächen werden frei für anderweitige Nutzung. Der Schlachtwert der Milchkühe nimmt ab!

Die Tendenz zu höheren Milchleistungen je Kuh wird sich aus ökonomischer Sicht noch weiter fortsetzen. In den Ländern der EU sind dennoch erhebliche Unterschiede im Anteil der Mutterkühe am Gesamtkuhbestand festzustellen (Tabelle 3).

In diesem Rahmen verdient Frankreich besondere Beachtung, weil hier neben dem hohen Anteil an reinrassigen Fleischrindern auch deren Qualität weltbekannt ist. Deutschland hat diesbezüglich keine Tradition und auch nur einen geringen Anteil. Die unserem Land durch die EU zugebilligten Quoten sind Ausgeschöpft und demzufolge stagniert nunmehr die quantitative Entwicklung der Mutterkuhbestände, die primär Kreuzungsprodukte sind.

Alle Länder Europäischen Union sind jedoch in der Rindfleischerzeugung mehr oder weniger durch die BSE - Krise betroffen worden.

Der Rindfleischverbrauch ging zurück, es fielen die Marktpreise, die Futtermittel wurden teurer und in der Verarbeitung traten durch die Beseitigung des Risikomaterials und die notwendigen BSE - Tests erhöhte Kosten auf.

Den Rückgang der Nachfrage infolge der BSE bei Frischfleisch in Deutschland verdeutlich die folgende Tabelle (Tabelle 4).

Gestiegen ist in dieser Zeit die Nachfrage nach Geflügel.
Der Pro - Kopf - Verbrauch betrug 1999 in Deutschland 15,2 kg. In den USA, dem Land mit der größten Rindfleischproduktion der Welt sehen die Produktions- und Verbrauchszahlen wie folgt aus (Tabelle 5).

Auch wenn wir weiter einen langsamen Anstieg der Nachfrage nach Rindfleisch beobachten, so sind die amerikanischen Zahlen nur von theoretischem Interesse. Sie machen uns aber Hoffnung auf bessere Zeiten als wir sie jetzt erleben.

Die Umstände zwingen uns aber im Interesse der Wirtschaftlichkeit den Qualitätsfragen bei der Erzeugung von Rindfleisch ein besonderes Augenmerk zu widmen. Das beginnt bereits mit der Auswahl der geeignetsten Rassen aus dem Potential der Fleischrinderpopulationen.

In $\mathrm{M} / \mathrm{V}$ ist nach der Wende mit einer großen Rassenvielfalt begonnen worden. Das zeigt Tabelle 6.

Allmählich bilden sich Schwerpunkte heraus von denen ich besonders das Charolais - Rind, die Fleckviehrasse und deren Kreuzungsprodukte „Uckermärker“ befürworte. Beste Ausschlachtungsresultate erreichen auch die Limousin - Tiere.

Bei der Beurteilung gehe ich sowohl von dem Mastleistungsvermögen, gemessen an den täglichen Zunahmen als auch vom Verkauf der Absetzer für den Export aus, wie er von vielen unserer größeren Betriebe praktiziert wird.

Im EUROP - System der Klassifizierung muss ein hoher Anteil der Schlachttiere die Aussicht auf eine Qualifizierung als $U$ und $R$ Schlachtkörper erreichen.

Zugeständnisse an die Rasse kann man bei nur extensiven Mastmöglichkeiten bzw. bei einer Direktvermarktung in der Nähe von Großstädten machen.

Meine diesbezüglichen Aussagen sollen auch durch die Angaben über die Leistungen von Spitzenbullen verschiedener Rassen untermauert werden (Tabelle 7).

Hier stehen die Charolais -, Fleckvieh - und Uckermärker Bullen sowohl mit ihren Lebenstageszunahmen als auch mit den für den Typ (T), die Bemuskelung (B) und dem Skelett (S) erhaltenen Bewertungen an der Spitze.

Zur Klärung der Frage nach den geeignetsten Rassen wurden in den verschiedenen Ländern umfangreiche Versuche angestellt. Einige Ergebnisse enthält die folgende Tabelle 8. 
Sie zeigt die in den Versuchen erhaltenen Rangfolgen bei dem ökonomisch wichtigem Merkmal „Masttagszunahme“.

In jedem Fall liegen die großrahmigen Rassen mit in der Spitzengruppe.

Ich weiß, dass in Polen ähnliche Versuche mit vergleichbaren Resultaten durchgeführt wurden in die auch die Salers einbezogen wurden. Die großrahmigen Typen eignen sich auch besonders für Kreuzung mit Charolais.

Für eine intensive Mast kann hieraus die Empfehlung für die Rassen Charolais und Simmentaler abgeleitet werden.

In der nachfolgenden Tabelle sind eine Reihe von Merkmalen bewertet, die in der Rindfleischerzeugung wichtig sind (Tabelle 9).

Hier ist hervorzuheben, dass die rahmigeren Rassen auch in der Schlachtausbeute gute Leistungen bringen. Es fallen die Piemontester diesbezüglich besonders vorteilhaft auf und auch die Limousin. In den größeren Mutterkuhherden unserer EZG mussten wir leider erhebliche Charakterschwächen bei Limousin feststellen, die auf ihr Temprament zurückzuführen sind. Bei individuellerer Betreuung, wie das in kleineren Herden gegeben ist, bereiten die Tiere keine größeren Schwierigkeiten.

Erkennbar ist auch die negative Korrelation zwischen dem Kaliber der Tiere und dem Kalbeverhalten. Diesbezüglich sind die BB als Extremrasse zu bezeichnen, bei der größte Teil der Kälber aus Färsen nach Schnittentbindung geboren werden.

Diese Tatsache schließt ihre Haltung in unserer EZG aus.

In den neuen Bundesländern erfolgte der Aufbau der Mutterkuhherden hauptsächlich mit weiblichen Jungrindern aus der Kreuzung Milchrind $\mathrm{x}$ Fleischrind. Im großen Umfang wurden auch Milchrindtypen der Rasse Fleckvieh (Simmentaler) aus Tschechien für diesen Zweck importiert.

Bei den Kreuzungsprodukten aus Milchrind $\mathrm{x}$ Fleischrind kann man mit folgenden Überlegenheiten bei Mastleistungen rechnen (Tabelle 10).

Die Mutterkühe aus den Kreuzungen zeichnen sich durch eine höhere Milchleistung zum Vorteil ihrer Saugkälber aus.

Bei der Bewirtschaftung der Mutterkuhherden ist in vielen Betrieben ein fester Jahresrhythmus eingeführt worden (Tabelle 11).

Als Beispiel möchte ich den Rhythmus mit einer Dezemberkalbung darstellen. Er bietet günstige Voraussetzungen für die Kälber in der Weidenutzung. Die Saugperiode kann lange ausgedehnt werden, bringt hohe Zunahmen bei viel wirtschaftseigenem Grünfutter, vor allem aber durch die separate Konzentratzufütterung an die Kälber.

In den größeren Beständen und bei einfachster Haltungsform der Kühe in Laufställen bringt die Kalbung im Winter oftmals eine erhöhte Infektionsgefahr für die Kälber mit sich und das hat überdurchschnittlich hohe Kälberverluste zur Folge. Viele Betriebe versuchen dem durch Mutterschutzimpfungen zu entgehen. Die meisten
Betriebe in unserer EZG weichen mit ihrer Kalbezeit auf die Frühjahrskalbung im März/April aus mit schneller Umstellung auf die Weide. Nicht wenige betreibe halten ihre Mutterkuhherden ganzjährig auf Weiden ohne Stall. Das erfordert natürlich die rechtzeitige Schaffung geschützter warmer Liegeplätze, die kontinuierlich mit Stroh eingestreut werden.

Die ganzjährige Weidehaltung verringert nicht nur die Infektionsgefahr, sie wirkt sich auch günstig auf das Kalbeverhalten der Tiere aus, wie wir mit Untersuchungsergebnissen mit Färsen nachweisen können (Tabelle 12).

Die nun folgende Tabelle 13 bringt Resultate aus größeren Herden mit verschiedenen Kalbezeiten. Es ist erkennbar, dass die Gesamtabgänge an Kälbern bei der Frühjahrskalbung am geringsten sind.

In kleineren Herden mit weitgehender individueller Betreuung können die Resultate anders ausfallen. So bevorzugen die Besitzer von Zuchtherden in Frankreich z. B. die November/Dezember Kalbung und demonstrieren damit auf ihren September - Schauen ausgezeichnete Produkte.

Im Rahmen unserer Untersuchungen ergaben sich aber bereits signifikante Differenzen zwischen den im Mai und im Juli geborenen Saugkälbern in der täglichen Zunahme (Tabelle 14).

Die Tabelle zeigt auch das erwartete Ergebnis in den Zunahmen der Tiere Verschiedenen Geschlechts.

Signifikant sind aber auch die Differenzen in den Zunahmen von Tieren aus Normal - und Schwergeburten bei bis zu 100 Lebenstagen.

Die Tabelle zeigt auch, dass man Färsen nicht mit zu geringem Lebengewicht zulassen sollte.

Wiegen sie nach der Kalbung weniger als 400 kg, dann nehmen die Kälber deutlich weniger zu als die aus schwereren Müttern.

Aber auch die über die Wetterverhältnisse und damit über die Vegetation wirkenden Einflüsse können, wie in der Tabelle gezeigt wird von statistisch gesicherter Größe sein.

Einige Ausführungen noch zu der in der Tabelle oben angeführten Säugedauer. Natürlich soll eine Mutterkuh so lange wie möglich ihr Kalb bei Fuß behalten. Wir bevorzugen Kreuzungskühe mit Fleckviehgenen wegen der höheren Milchleistung. Eine Säugedauer von mindestens 5-6 Monaten wirkt sich günstig auf das Wachstum der Kälber aus und erleichtert den Übergang zum Festfutter. Warum sollte auch eine Mutterkuh nur kurzzeitig in der Laktation genutzt werden? Allerdings muss man bei den mit viel Milch ernährten weiblichen Kälbern schon nach 5 Lebensmonaten mit der Geschlechtsreiferechnen rechnen.

Eine rechtzeitige Geschlechtertrennung ist deshalb anzuraten oder die Trächtigkeit wird mit vet. med. Mitteln-abgebrochen.

Das ökonomische Ergebnis der Muterkuhhaltung wird ganz wesentlich durch die Trächtigkeitsrate mit bestimmt. Auf die Konzeptionsrate können eine Reihe von Faktoren wirken. Wir haben das an einer 
großen Zahl von Kreuzungsfärsen untersucht (Tabelle 15).

An der Resultaten wird deutlich, dass der Genotyp der Rinder dabei von geringerer Bedeutung ist. In großen Herden ist aber der Einsatz von Herdenbullen unumgänglich.

Die Tabelle zeigt aber auch, dass die Beachtung des Konzeptionsalters und des Gewichtes der Tiere zu diesem Zeitpunkt von beachtlichem Einfluss sein können.

Besonders schlecht sind die Konzeptionsraten bei sehr jungen und zu leichten Rindern. Natürlich kann man die Grenzen durch die Fütterungsintensität variieren.

In den Mutterkuhherden kann der Arbeitsaufwand ganz wesentlich durch den Geburtsverlauf bei den Kalbinnen beeinflusst werden, wie die Tabelle 16 zeigt.

Die Normalgeburtenrate (NGR) steigt mit zunehmendem Kalbealter und höherem Lebendgewicht. Sie nimmt aber beträchtlich ab, wenn das relative Geburtsgewicht der Kälber zunimmt. Das hiermit verbundene Risiko kann durch den Einsatz geprüfter Bullen gemindert werden. Für die Belegung von Färsen vorgesehene Deckbullen sollten zuvor an Kühen getestet sein. Bekanntlich ist das Geburtsgewicht der Kälber auch genetisch bedingt, so dass beim Bulleneinsatz nicht nur die Rassezugehörigkeit, sondern auch die individuelle Eignung der Väter innerhalb der Rassen beachtet werden muss. Je vermehrt sogenannte Absetzer erzeugt.

Das sind Tiere, die in der Regel als Bullen etwa $250 \mathrm{~kg}$ schwer sind und dieses Gewicht in ungefähr 6 Monaten erreicht haben. Ihre weiblichen Altersgefährten kommen in derselben Zeit auf annähernd $210 \mathrm{~kg}$.
Die Tiere gehen in Mastbetriebe des In - und Auslandes. Die Abnehmer bevorzugen Jungrinder mit weißer Farbe und zahlen dafür den höchsten Preis. Die Farbe ist für sie der Hinweis auf die Charolais - Gene und damit ein „Markenzeichen“ für gute Mastfähigkeit. Dunkel gefärbte Absetzer, bei denen die Farbe auf weniger gut bemuskelte und kleinrahmige Rassen schließen lässt, finden über diesen Markt kaum Interessenten bzw. sind mit ihnen nur unbefriedigende Preise zu erzielen.

Die gute Eignung der Charolais - Genträger für die Mast bestätigen auch unsere Mitgliedsbetreibe. Bei den Einstufungen auf den Schlachthöfen nach dem EUROP- System schneiden sie am besten ab.

Sie haben bei intensiver Mast berechtigte Aussichten in die Handelsklassen U und R eingestuft zu werden und damit die jeweils aktuell besten Preise zu erzielen.

Nach der BSE - Krise hat die Orientierung auf eine ökologisch ausgerichtete Erzeugung an Nahrungsmitteln zugenommen. Betreibe unserer EZG produzieren nach wie vor konventionell und über die Mutterkuhhaltung qualitative hochwertige Erzeugnisse.

Diese konventionelle Erzeugung wird auch in Zukunft dominieren. Die Produktion kann durch entsprechend anerkannte Instanzen begutachtet und für den Handel zertifiziert werden. Die vollständig auf die ökologische Erzeugung ausgerichteten Betriebe produzieren teurer und müssen ihre Erzeugnisse entsprechend teurer vermarkten können. Dafür fehlt besonders in den neuen Bundesländern oftmals noch die Kaufkraft.

Beide Formen werden aber nebeneinander bestehen. (1.000St.)

\begin{tabular}{|c|c|c|c|c|c|c|}
\hline \multirow{2}{*}{ Tierart } & \multirow{2}{*}{$\begin{array}{l}\text { Dez. } \\
1991\end{array}$} & \multicolumn{3}{|c|}{ November } & \multicolumn{2}{|c|}{ Entwicklung \% } \\
\hline & & 1998 & 1999 & 2000 & 00:99 & 00:91 \\
\hline Rinder gesamt & 730,9 & 595,8 & 590,2 & 573,6 & 97,2 & 78,5 \\
\hline dar. Milchkühe & 248,4 & 204,0 & 193,2 & 185,8 & 96,2 & 74,8 \\
\hline dar. Mutterkühe & 11,5 & 61,8 & 73,3 & 76,0 & 103,7 & 660,9 \\
\hline
\end{tabular}

Tabelle 2

Durchschnittliche Milchleistung aller geprüften Kühe 2001 (vorläufig)

\begin{tabular}{|l|r|r|r|}
\hline \multicolumn{1}{|c|}{ Land } & $\begin{array}{c}\text { Milch } \\
\text { kg }\end{array}$ & $\begin{array}{c}\text { Fett } \\
\text { \% }\end{array}$ & \multicolumn{1}{c|}{$\begin{array}{c}\text { Eiweiß } \\
\text { \% }\end{array}$} \\
\hline Mecklenburg - Vorpommern & 7.675 & 4,20 & 3,45 \\
\hline Brandenburg & 7.616 & 4,15 & 3,47 \\
\hline Sachsen - Anhalt & 7.745 & 4,18 & 3,46 \\
\hline Thüringen & 7.559 & 4,19 & 3,41 \\
\hline Sachsen & 7.629 & 4,26 & 3,47 \\
\hline Deutschland 2001 & 7.132 & 4,21 & 3,43 \\
\hline
\end{tabular}

Anteil Mutterkühe

in Prozent der insgesamt gehaltenen Kühe

\begin{tabular}{|l|r|}
\hline Spanien & $60 \%$ \\
\hline Frankreich & $51 \%$ \\
\hline Irland & $49 \%$ \\
\hline Portugal & $45 \%$ \\
\hline Ver. Königreich & $44 \%$ \\
\hline Schweden & $28 \%$ \\
\hline Italien & $22 \%$ \\
\hline Dänemark & $17 \%$ \\
\hline Deutschland & $15 \%$ \\
\hline Finnland & $7 \%$ \\
\hline Niederlande & $6 \%$ \\
\hline
\end{tabular}


Private nachfrage bei Frischfleisch

Veränderung der Einkaufsmengen in \% (im Vergleich zum Vorjahreszeitraum)

\begin{tabular}{|c|c|c|c|c|c|}
\hline & $\begin{array}{c}\text { 25.11. bis } \\
31.12 .2000\end{array}$ & $\begin{array}{l}\text { 1. Quartal } \\
2001\end{array}$ & $\begin{array}{l}\text { 2. Quartal } \\
2001\end{array}$ & $\begin{array}{l}\text { 3. Quartal } \\
2001\end{array}$ & $\begin{array}{c}\text { Prognose } \\
2001\end{array}$ \\
\hline Rind & -68 & -58 & -29 & -26 & -25 \\
\hline Schwein & -4 & 2 & -1 & -2 & 0 \\
\hline Rind/Schwein gem. & -55 & -49 & -23 & -16 & -19 \\
\hline Geflügel & 8 & 10 & 5 & -2 & 7 \\
\hline
\end{tabular}

Tabelle 5

Produktion und Verbrauch

von Rind - und Kalbfleisch in den USA

\begin{tabular}{|l|r|r|r|r|r|r|}
\hline & \multicolumn{1}{|c|}{$\mathbf{1 9 9 6}$} & \multicolumn{1}{c|}{$\mathbf{1 9 9 7}$} & \multicolumn{1}{c|}{$\mathbf{1 9 9 8}$} & \multicolumn{1}{c|}{$\mathbf{1 9 9 9}$} & \multicolumn{1}{c|}{$\mathbf{2 0 0 0}$} & $\mathbf{2 0 0 1}$ \\
\hline $\begin{array}{l}\text { Produktion } \\
\text { (in. 1.000 t) }\end{array}$ & 11.749 & 11.714 & 11.804 & 12.124 & 12.298 & 11.752 \\
\hline $\begin{array}{l}\text { Verbrauch } \\
\text { (in 1.000 t) }\end{array}$ & 11.903 & 11.767 & 12.052 & 12.324 & 12.481 & 12.053 \\
\hline $\begin{array}{l}\text { Pro - Kopf - Verbrauch } \\
\text { (in kg) }\end{array}$ & 44,9 & 43,9 & 44,6 & 45,2 & 45,4 & 43,5 \\
\hline
\end{tabular}

Tabelle 6

Entwicklung der Fleischrind - Herdbuchbestände in Mecklenburg - Vorpommern

\begin{tabular}{|l|r|r|r|r|}
\hline \multirow{2}{*}{ Rasse } & \multicolumn{2}{|c|}{ Bullen in Stück } & \multicolumn{2}{c|}{ Kühe in Stück } \\
\cline { 2 - 5 } & \multicolumn{1}{c|}{$\mathbf{1 9 9 1}$} & \multicolumn{1}{c|}{$\mathbf{1 9 9 9}$} & $\mathbf{1 9 9 1}$ & \multicolumn{1}{c|}{$\mathbf{1 9 9 9}$} \\
\hline Angus & 6 & 88 & 83 & 2.169 \\
\hline Aubrac & - & 9 & - & 90 \\
\hline Blonde d Aquitaine & - & 1 & - & 1 \\
\hline Charolais & 16 & 128 & 190 & 1.075 \\
\hline Chianina & - & - & 4 & - \\
\hline Fleckvieh & 31 & 231 & 330 & 7.676 \\
\hline Galloway & 17 & 53 & 203 & 424 \\
\hline Gelbvieh & - & 4 & - & 111 \\
\hline Hereford & 5 & 38 & 98 & 527 \\
\hline Highland & 2 & 35 & 13 & 183 \\
\hline Limousin & 16 & 114 & 181 & 687 \\
\hline Piemonteser & - & - & - & - \\
\hline Pinzgauer & - & 7 & - & 486 \\
\hline Salers & - & 36 & - & 424 \\
\hline South - Devon & - & - & - & 5 \\
\hline Shorthorn & 1 & 2 & - & 17 \\
\hline Uckermärker & 13 & 23 & - & 192 \\
\hline Welsh Black & 3 & 3 & 38 & 27 \\
\hline Gesamt: & $\mathbf{1 1 0}$ & $\mathbf{7 7 2}$ & $\mathbf{1 . 1 3 6}$ & $\mathbf{1 4 . 0 9 4}$ \\
\hline
\end{tabular}

Tabelle 7

Spitzenergebnisse der Eigenleistungsprüfung von

Fleischrindbullen im Feld

$(10 / 99-09 / 2000)$

\begin{tabular}{|l|l|r|r|r|r|}
\hline \multirow{2}{*}{ Rasse } & \multirow{2}{*}{ Name } & LTZ & \multicolumn{3}{|c|}{ Bewertung } \\
\cline { 4 - 6 } & & g & T & \multicolumn{1}{c|}{ B } & \multicolumn{1}{c|}{ S } \\
\hline Deutsche Angus & Veit & 1.477 & 7 & 8 & 6 \\
\hline Deutsche Angus & Hagen & 1.333 & 7 & 8 & 7 \\
\hline Deutsche Angus & Ronny & 1.300 & 7 & 8 & 7 \\
\hline Charolais & Goliath & 1.618 & 8 & 8 & 8 \\
\hline Charolais & Adam & 1.524 & 8 & 8 & 7 \\
\hline Charolais & Ramses & 1.519 & 7 & 8 & 6 \\
\hline Fleckvieh & Jaros & 1.656 & 8 & 8 & 8 \\
\hline Fleckvieh & Korus & 1.599 & 8 & 8 & 7 \\
\hline Fleckvieh & Heldor & 1.542 & 8 & 8 & 8 \\
\hline Hereford & Danny & 1.225 & 8 & 7 & 7 \\
\hline Limousin & Wilhelm & 1.412 & 7 & 9 & 7 \\
\hline Limousin & Waldemar & 1.379 & 8 & 8 & 8 \\
\hline Limousin & Flox & 1.254 & 7 & 7 & 7 \\
\hline Salers & Oliver & 1.405 & 7 & 8 & 7 \\
\hline Salers & Olaf & 1.275 & 7 & 7 & 7 \\
\hline Uckermärker & Ficus & 1.500 & 8 & 8 & 7 \\
\hline Uckermärker & Findus & 1.408 & 7 & 7 & 7 \\
\hline
\end{tabular}


Masttagszunahme

\begin{tabular}{|c|c|c|c|c|c|c|c|}
\hline \multicolumn{2}{|c|}{ Eigene Ergebnisse } & \multicolumn{2}{|c|}{ Dänemark } & \multicolumn{2}{|c|}{ Frankreich } & \multicolumn{2}{|c|}{$\begin{array}{c}\text { USA } \\
\text { 3) }\end{array}$} \\
\hline Rasse & g/d & Rasse & g/d & Rasse & g/d & Rasse & g/d \\
\hline Ro & 1022 & Cha & 990 & Cha & 1213 & Ма & 1160 \\
\hline FL & 99,6 & $\mathrm{Si}$ & 99,0 & Chi & 98,3 & $\mathrm{Si}$ & 99,6 \\
\hline Cha & 99,4 & $\mathrm{BdA}$ & 98,9 & Ma & 98,0 & Cha & 98,8 \\
\hline Chi & 99,1 & Ro & 98,4 & $\mathrm{He}$ & 97,5 & GV & 96,2 \\
\hline Ма & 97,1 & Chi & 96,7 & SD & 97,3 & SD & 95,6 \\
\hline $\mathrm{BB}$ & 97,6 & $\mathrm{BB}$ & 96,4 & $\mathrm{Si}$ & 97,0 & Chi & 93,4 \\
\hline SD & 95,0 & $\mathrm{He}$ & 93,3 & $\mathrm{BB}$ & 96,5 & $\mathrm{Li}$ & 86,0 \\
\hline $\mathrm{Pi}$ & 95,6 & GV & 92,3 & $\mathrm{Pi}$ & 95,9 & & \\
\hline $\mathrm{Li}$ & 92,2 & SD & 91,8 & $\mathrm{BdA}$ & 95,8 & & \\
\hline $\mathrm{He}$ & 91,1 & $\mathrm{Li}$ & 91,6 & $\mathrm{Li}$ & 93,7 & & \\
\hline & & $\mathrm{Pi}$ & 90,8 & & & & \\
\hline SR & 94,8 & $\mathrm{RD} / \mathrm{DF}$ & 96,6 & $\mathrm{~F}$ & 90,7 & $\mathrm{He} / \mathrm{AA}$ & 88,4 \\
\hline
\end{tabular}

1) $50 .-450 . \mathrm{LT}$

2) 238.-481. LT

3) 200./225.-405./424.LT

Tabelle 9

Eigenschaften ausgewählter Fleisch - und fleischbetonter Zweinutzungsrassen

\begin{tabular}{|c|c|c|c|c|c|c|c|c|c|c|c|}
\hline & Cha & Lim & BA & FV & GV & $\mathbf{P i}$ & BB & DA & AA & He & Hl \\
\hline Gewicht und Rahmen & +++ & ++ & +++ & +++ & +++ & ++ & +++ & ++ & + & + & - \\
\hline Muskulatur & +++ & +++ & +++ & ++ & + & +++ & +++ & + & + & + & - \\
\hline Feine Knochen & - & +++ & +++ & + & + & +++ & +++ & ++ & +++ & + & ++ \\
\hline LMZ & +++ & ++ & +++ & +++ & ++ & ++ & +++ & ++ & + & + & - \\
\hline Schlachtausbeute & +++ & +++ & +++ & ++ & +++ & +++ & +++ & ++ & + & + & - \\
\hline Charakter & +++ & + & +++ & +++ & +++ & +++ & +++ & + & + & + & +++ \\
\hline Milchleistung & ++ & + & + & +++ & ++ & ++ & + & + & + & + & - \\
\hline Abkalbung & + & +++ & ++ & ++ & +++ & ++ & - & ++ & +++ & +++ & +++ \\
\hline Fruchtbarkeit & +++ & +++ & + & +++ & & ++ & - & +++ & +++ & +++ & +++ \\
\hline
\end{tabular}

$+++=$ sehr gut, $\quad++=$ gut, $+=$ ausreichend, $\quad-=$ nicht ausreichend

Tabelle 10

Vorteile von Tieren aus der Gebrauchskreuzung (Rind - MR $\times$ FR)

\begin{tabular}{|l|r|}
\hline \multicolumn{1}{|c|}{ Kennwert } & MR = 100 \\
\hline MTZ (Masttagszunahme) & $+\mathbf{8}-\mathbf{1 0}$ \\
\hline SA (Schlachtausbeute) & $+2-3$ \\
\hline Anteil hochwertiger Teilstücke & $+2-4$ \\
\hline Futter - Aufwand & $-5-10$ \\
\hline
\end{tabular}

Tabelle 12

Schwergeburtenanteil bei Färsen in Abhängigkeit von der Haltungsform

\begin{tabular}{|c|c|c|c|c|}
\hline \multirow[t]{2}{*}{ Betrieb } & \multicolumn{2}{|c|}{ Weidehaltung } & \multicolumn{2}{|c|}{ Stallhaltung } \\
\hline & $\begin{array}{c}\text { Geburten } \\
\mathrm{n}\end{array}$ & $\begin{array}{c}\text { Schwer- } \\
\text { geburten } \\
\%\end{array}$ & $\begin{array}{c}\text { Geburten } \\
\mathrm{n}\end{array}$ & $\begin{array}{c}\text { Schwer- } \\
\text { geburten } \\
\%\end{array}$ \\
\hline $\begin{array}{l}\text { mehrere } \\
\text { Betriebe }\end{array}$ & 438 & 7,8 & 234 & 31,2 \\
\hline Betrieb & 396 & 8,1 & - & - \\
\hline Gutens- & 413 & 4,6 & 156 & 17,2 \\
\hline wegen & 555 & 6,7 & - & - \\
\hline
\end{tabular}

Tabelle 11 Jahresrhythmus in der Mutterkuhhaltung am Beispiel der Dezemberkalbung

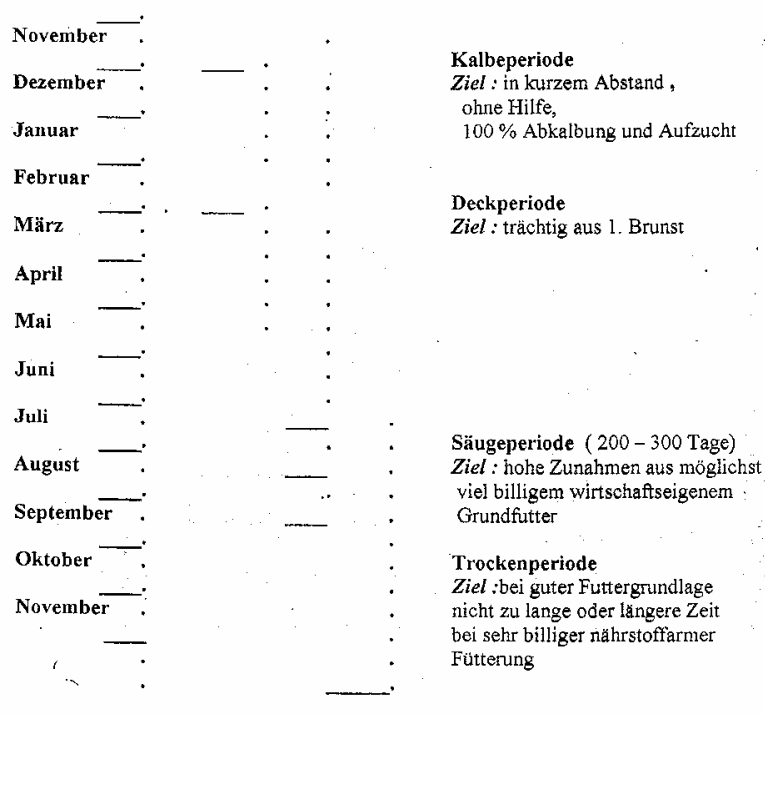


Verluste, Notschlachtungen und Gesamtabgänge von Kalbung bis zum Absetzen

\begin{tabular}{|c|c|c|c|c|c|c|c|}
\hline \multirow[t]{2}{*}{ Abkalbgruppe } & \multirow{2}{*}{$\begin{array}{c}\text { Abkalbende } \\
\text { Kühe } \\
\mathbf{n} \\
\end{array}$} & \multicolumn{2}{|c|}{$\begin{array}{c}\text { Davon } \\
\text { leb. geb. Kälber }\end{array}$} & \multirow{2}{*}{$\begin{array}{c}\text { Totgeburten } \\
\% \\
\end{array}$} & \multirow{2}{*}{$\begin{array}{c}\begin{array}{c}\text { Verluste von } \\
\text { leb.geb. } \\
\text { Kälbern }\end{array} \\
\% \\
\end{array}$} & \multirow{2}{*}{$\begin{array}{c}\text { Notschlach- } \\
\text { tungen v. leb. } \\
\text { Geb. } \\
\text { Kälbern } \\
\% \\
\end{array}$} & \multirow{2}{*}{$\begin{array}{c}\text { Abgänge ges. } \\
\text { v Kalbung } \\
\text { bis Absetzen } \\
\% \\
\end{array}$} \\
\hline & & $\mathbf{n}$ & $\%$ & & & & \\
\hline Dez.-Jan. & 102 & 100 & 98,0 & 2,0 & 5,0 & 3,0 & 9,8 \\
\hline März-April & 81 & 80 & 98,8 & 1,2 & 2,5 & 2,5 & 6,2 \\
\hline Mai-Juni & 422 & 418 & 99,1 & 0,9 & 4,5 & 1,2 & 6,6 \\
\hline Sept.-Okt. & 113 & 111 & 98,2 & 1,8 & 6,0 & 3,6 & 11,5 \\
\hline gesamt & 718 & 709 & 98,7 & 1,3 & 4,7 & 2,0 & 7,8 \\
\hline
\end{tabular}

Einfluss ausgewählter Faktoren auf die mittleren Tageszunahmen / TZN (g) von Saugkälbern

\begin{tabular}{|c|c|c|c|c|}
\hline \multicolumn{2}{|c|}{ Einflussfaktoren } & \multirow{2}{*}{$\begin{array}{l}\mathbf{n} \\
\end{array}$} & \multirow{2}{*}{\begin{tabular}{ll|} 
TZN & \\
& 942 \\
\end{tabular}} & \multirow{2}{*}{$\begin{array}{c}\text { Signif. } \\
++\end{array}$} \\
\hline Säugedauer & $>100$ & & & \\
\hline (Tage) & $<80$ & 452 & 843 & \\
\hline \multirow[t]{2}{*}{ Kalbemonat } & Mai & 281 & 920 & ++ \\
\hline & Juli & 357 & 850 & \\
\hline \multirow[t]{2}{*}{ Geschlecht der Kälber } & mannlich & 627 & 907 & ++ \\
\hline & weiblich & 582 & 850 & \\
\hline \multirow[t]{2}{*}{ Geburts - verlauf } & normal & 1201 & 884 & ++ \\
\hline & schwer & 65 & 778 & \\
\hline \multirow{2}{*}{$\begin{array}{l}\text { Lebendmasse der } \\
\text { Mutter p - p (kg) }\end{array}$} & 451 bis 500 & 332 & 910 & ++ \\
\hline & $<400$ & 272 & 821 & \\
\hline \multirow[t]{2}{*}{ Jahrgang } & I & 423 & 914 & ++ \\
\hline & II & 459 & 824 & \\
\hline
\end{tabular}

Tabelle 15

Konzeptionsrate (KR \%) bei Kreuzungsfärsen in Abhängigkeit von ausgewählten Einflussfaktoren

\begin{tabular}{|l|r|r|}
\hline \multicolumn{1}{|c|}{ Einflussfaktoren } & $\begin{array}{r}\text { Anzahl der } \\
\text { Mastfärsen }\end{array}$ & \multicolumn{1}{c|}{ KR \% } \\
\hline Genotyp der Färse & 1990 & \\
\hline Fleischfleckvieh $\times$ MR & 243 & 82,4 \\
FF $\times$ Cha $\times$ MR & & 80,4 \\
\hline Belegungsart & 879 & \\
künstl. Besamung & 2963 & 40,7 \\
Herdenbulle & & 83,8 \\
\hline Konzeptionsalter (Mon.) & 360 & \\
bis $\quad \mathbf{1 4}$ & 471 & 60,0 \\
$\mathbf{1 5}$... $\quad \mathbf{1 7}$ & 321 & 80,7 \\
$\mathbf{1 8}$... $\quad \mathbf{2 0}$ & & 87,2 \\
\hline Konzeptionsalter (kg.) & 156 & \\
bis $\quad \mathbf{2 8 0}$ & 459 & 55,1 \\
$\mathbf{3 2 1}$... $\mathbf{3 6 0}$ & 281 & 85,4 \\
$\mathbf{3 6 1}$... $\quad \mathbf{4 0 0}$ & & 87,2 \\
\hline
\end{tabular}

Tabelle 16 Prozentuale Angaben zum Geburtsverlauf (GV 1 ... 6 = 100\%) bei Kreuzungsfärsen

\begin{tabular}{|l|r|r|r|r|}
\hline \multicolumn{1}{|c|}{ Einflussfaktor } & \multirow{2}{*}{$\mathbf{n}$} & \multicolumn{1}{c|}{ GV } & GV & \multirow{2}{*}{ NGR } \\
\cline { 3 - 4 } & & $\mathbf{2}$... 5 & \multicolumn{1}{c|}{$\mathbf{5}$} & \\
\hline Genotyp der Mutter & & & & \\
\hline Fleischfleckvieh x MR & 1283 & 8,0 & 2,3 & 92,0 \\
\hline FF x Cha x MR & 137 & 8,8 & 2,9 & 91,2 \\
\hline Kalbealter & & & & \\
\hline bis 24 Monate & 118 & 17,0 & 6,8 & 81,6 \\
\hline 27 ... 30 Monate & 354 & 7,1 & 1,7 & 92,7 \\
\hline Lebendmasse p.p. & & & & \\
\hline bis 400 kg & 318 & 13,2 & 4,7 & 86,2 \\
\hline ca. 51 ... 500 kg & 365 & 5,6 & 1,4 & 94,1 \\
\hline rel. Geburtsmasse d. Kälber & & & & \\
\hline bis 6,9\% & 160 & 1,2 & - & 98,7 \\
\hline 8,0 ... 8,9\% & 421 & 6,2 & 1,9 & 93,6 \\
\hline ab 9,0\% & 456 & 16,9 & 5,3 & 82,7 \\
\hline
\end{tabular}

